\title{
Time-Series Predictability in the Disaster Model
}

\author{
François Gourio*
}

First Version: December 2007

This Version: August 2008

\begin{abstract}
This paper studies whether the Rietz-Barro "disaster" model, extended for a time-varying probability of disaster, can match the empirical evidence on predictability of stock returns. It is shown that when utility is CRRA, the model cannot replicate these findings, regardless of parameter values. This motivates extending the disaster model to allow for Epstein-Zin utility. Analytical results show that when the probability of disaster is iid, the model with Epstein-Zin utility can match the evidence on predictability qualitatively if the elasticity of substitution is greater than unity. The case of a persistent probability of disaster is studied numerically, with partial success.
\end{abstract}

\section{Introduction}

There has been lately a revival of interest in the "disaster" explanation of asset prices, which was first introduced by Rietz (1988). Rietz showed that the possibility of rare jumps in consumption, can solve the equity premium puzzle of Mehra and Prescott (1985). Barro (2006) measures disasters during the XXth century, and he finds that they are frequent and large enough, and stock returns low enough relative to bond returns during disasters, to make the explanation quantitatively plausible.

Given the success of the disaster model in accounting for the risk-free rate and equity premium puzzles, it is important to study if the model can also account for additional asset pricing facts. In this note I focus on the time-series predictability of stock returns and excess stock returns. Empirical research documents that both the stock return and the excess stock return are forecastable. The basic regression is:

$$
R_{t+1}^{e}-R_{t+1}^{f}=\alpha+\beta \frac{D_{t}}{P_{t}}+\varepsilon_{t+1},
$$

where $R_{t+1}^{e}$ is the equity return, $R_{t+1}^{f}$ the risk-free return, and $\frac{D_{t}}{P_{t}}$ the dividend yield. As an illustration, John Cochrane (2008) reports for the annual 1926-2004 U.S. sample: $\beta=3.83$ (t-stat $=2.61, R^{2}=$ $7.4 \%$ ). A key feature of the data is that using as the left-hand side the equity return $R_{t+1}^{e}$ rather than the excess return $R_{t+1}^{e}-R_{t+1}^{f}$ does not change the results markedly: $\beta=3.39\left(\mathrm{t}\right.$-stat $=2.28, R^{2}=5.8 \%$ )

*I thank participants in presentations at the AEA meetings, Clemson University, the Green Line Macro Meetings, and Northwestern (Kellog) business school for comments. I also thank John Campbell, Xavier Gabaix, Ian Martin and Adrien Verdelhan, and an anonymous referee for helpful comments. Contact information: Boston University, Department of Economics, 270 Bay State Road, Boston MA 02215. Email: fgourio@bu.edu. Tel.: (617) 3534534. 
That is, equity returns are forecastable, because risk premia are forecastable, and not because of an important predictable component in the risk-free interest rate. This note studies whether a reasonable extension of the disaster model can match these empirical findings. ${ }^{1}$

More precisely, this note extends the disaster model to incorporate a time-varying probability (or size) of disaster. The main result is that if utility is CRRA, the disaster model cannot account for both findings of stock return and excess stock return predictability. The intuition is that risk-free rates are too volatile in the model: a high probability of disaster reduces the risk-free rate more than it increases the equity risk premium. Or to put this in a different way, the variance of P-D ratios is too low in the model. Next, I show that analytically, in the case of i.i.d. shocks to the probability of disaster, that using Epstein-Zin utility with an elasticity of substitution larger than unity can resolve this puzzle. Finally, the more realistic case of Epstein-Zin utility and persistent changes in probability of disaster is analyzed through numerical simulations, with some success. However, one must assume that the probability of disaster is volatile and highly persistent to obtain an approximate quantitative match of the data. ${ }^{2}$

These results clarify and extend some findings of Xavier Gabaix (2007). Gabaix uses the "linearitygenerating" model (Gabaix 2007b), and expresses some of his results in terms of a "resilience" variable. Expected returns change over time because the probability of a disaster, the potential size of consumption disaster, or the potential size of dividend disaster changes over time, but my results show that only when the potential size of dividend disaster changes over time, and the size of consumption disaster doesn't, is the model (with power utility) consistent with the evidence on time-series predictability. Empirical research suggests that the expected return on many assets are correlated. In the consumption-based model, expected returns can be positively correlated across assets because they are all affected by properties of consumption (e.g., Campbell and Cochrane (1999) or Bansal and Yaron (2004)). But if variation in expected returns is due to variation in the potential size of dividend disaster, it is not clear why the expected returns should be correlated across assets.

Beside this substantive contribution of spelling out the properties and limitations of the disaster model, a technical contribution of the paper is to show a simple way in which to introduce time-varying risk premia and interest rates in the consumption-based model. Most models with time-varying interest rates and time-varying risk premia are untractable, and as a result they are usually analyzed numerically, or through the Campbell-Shiller approximations (e.g., Campbell (1996)), which assume joint conditional $\log$-normality.

The note is organized as follows. Section 2 analyzes the model with power utility, and section 3 considers the case of Epstein-Zin utility and i.i.d. disasters. These two sections offer analytical results. Section 4 provides some numerical simulations which illustrate the theoretical results and relax some

\footnotetext{
${ }^{1}$ It may seem strange to concentrate on these regression results, which are somewhat fragile, as shown by Stambaugh (1999), Ang and Bekaert (2007), Boudoukh, Richardson and Whitelaw (2006), and Goyal and Welch (2007) among others. The reason why I focus on these results is that the dividend yield (or similar measures of fundamentals scaled by price) is the most common predictor of returns. These results have motivated the development of models with time-varying risk premia such as Campbell and Cochrane (1999). Finally and most importantly, these results show that the volatility of the price-dividend ratio is largely due to the volatility of expected returns rather than dividends. Hence, matching this regression evidence is tantamount to tackling the stock-market volatility puzzle.

${ }^{2}$ The results of this paper are closely related to results in Wachter (2008), which was written simultaneously.
} 
of the assumptions, and finally considers the more promising case of Epstein-Zin utility and persistent shocks to the probability of disasters.

\section{Time-varying Disaster Probability with Power utility}

The model is a standard "Lucas tree" endowment economy. There is a representative consumer who has power utility (constant relative risk aversion):

$$
E \sum_{t=0}^{\infty} \beta^{t} \frac{C_{t}^{1-\gamma}}{1-\gamma}
$$

Following Barro (2006) and Rietz (1988), I assume that the main risk in the economy is a rare, large downward jump. To match the regression evidence outlined in the introduction, we need to generate variation in expected returns over time, which requires introducing some variation over time in the riskiness of the economy. The natural idea is to make the probability of disaster time-varying. For instance, the perceived risk of war certainly varies over time: it was higher during the Cold War than during the 1990s. (The case of a time-varying size of disasters is tackled below.) Assume, then, that the endowment follows the stochastic process:

$$
\begin{aligned}
\Delta \log C_{t+1} & =\mu+\sigma \varepsilon_{t+1}, \text { with probability } 1-p_{t} \\
& =\mu+\sigma \varepsilon_{t+1}+\log (1-b), \text { with probability } p_{t}
\end{aligned}
$$

where $\varepsilon_{t+1}$ is $\operatorname{iid} N(0,1)$ and $0<b<1$ is the size of the disaster. Hence, in period $t+1$, with probability $p_{t}$, consumption drops by a factor $b$. The disaster probability $p_{t} \in[\underline{p}, \bar{p}]$ evolves over time according to a first-order Markov process, governed by the transition probabilities $F\left(p_{t+1} \mid p_{t}\right)$. Note that $p_{t}$ is the probability of a disaster at time $t+1$, and it is drawn at time $t$. The Markov process is assumed to be monotone, i.e. $F\left(x \mid y_{1}\right) \leq F\left(x \mid y_{2}\right)$ for any $x \in[\underline{p}, \bar{p}]$ and for any $y_{1} \geq y_{2}$. This assumption means that $p_{t}$ is positively autocorrelated. The Markov process is also assumed to have the Feller property. ${ }^{3}$ The realization of the disaster, and the process $\left\{p_{t}\right\}$ are further assumed to be statistically independent of $\varepsilon_{t}$ at all dates. Finally, I assume that the realization of $p_{t+1}$ is independent of the realization of disasters at time $t+1$, conditional on $p_{t}$. That is, the new draw for the probability of disaster at time $t+2$, labelled $p_{t+1}$, is independent of whether there is a disaster at time $t+1$. This assumption implies that the P-D ratio is conditionally uncorrelated with current dividend growth or consumption growth, and hence the innovations to the state variable $p_{t}$ are not priced. ${ }^{4}$ This simplification is crucial to obtain analytical results. ${ }^{5}$ In Section 4 , I relax this assumption in numerical simulations and it seems to have a small quantitative impact.

\footnotetext{
${ }^{3}$ That is, the conditional expectation of a continuous function of the state tomorrow is itself a continuous function of the state today.

${ }^{4}$ In the data used by Cochrane (2008), the unconditional correlation between the dividend yield and dividend growth is .046 (s.e.: 0.111). The conditional correlation is .085 (s.e.: 0.119) if one uses the lagged dividend yield as conditioning variable.

${ }^{5}$ This assumption could be wrong either way: a disaster today may indicate that the economy is entering a phase of low growth or is less resilient than thought; but on the other hand, if a disaster occurred today, and GDP fell by $43 \%$, it is unlikely that GDP will fall again by a large amount. Rather, historical evidence suggests that the economy is likely to grow above trend for a while (Gourio (2008)).
} 
This simple economy has a single state variable, the probability of disaster $p$. We can express the asset prices as a function of this state variable, which is assumed to be perfectly observed by the agents in the economy. The gross risk-free rate $R_{t+1}^{f}$ satisfies the usual Euler equation:

$$
E_{t}\left(\beta\left(\frac{C_{t+1}}{C_{t}}\right)^{-\gamma}\right) R_{t+1}^{f}=1 .
$$

Computing this conditional expectation ${ }^{6}$ yields:

$$
\log R^{f}(p)=-\log \beta+\gamma \mu-\frac{\gamma^{2} \sigma^{2}}{2}-\log \left(1-p+p(1-b)^{-\gamma}\right)
$$

The risk-free rate varies over time with $p$. When $p=0$, this formula collapses to the well-known result of the i.i.d. lognormal model. Because $b<1$, we see that the risk-free rate is lower when $p>0$, and the higher the probability of a disaster, the lower the risk-free rate. This reflects that a higher probability of disaster reduces expected growth and increases risk, and thus leads agents to save, both for intertemporal substitution and for precautionary reasons. (However, the later are more important quantitatively.) This drives the risk-free rate down. ${ }^{7}$

The second asset we consider is a "stock", i.e. an asset which pays out the consumption process. The stock price satisfies the standard recursion:

$$
\frac{P_{t}}{C_{t}}=E_{t}\left(\beta\left(\frac{C_{t+1}}{C_{t}}\right)^{1-\gamma}\left(\frac{P_{t+1}}{C_{t+1}}+1\right)\right) .
$$

As usual, the price-dividend ratio depends only on the state variable, in this case $p$. Denote $q(p)$ the P-D ratio when $p_{t}=p$. Given the assumption that the realization of disaster is independent of the new draw for $p$, conditional on the current value of $p, q$ satisfies the functional equation:

$$
q(p)=\beta e^{(1-\gamma) \mu+(1-\gamma)^{2} \frac{\sigma^{2}}{2}}\left(1-p+p(1-b)^{1-\gamma}\right) \int_{\underline{p}}^{\bar{p}}\left(q\left(p^{\prime}\right)+1\right) d F\left(p^{\prime} \mid p\right) .
$$

Let $g(p)=\beta e^{(1-\gamma) \mu+(1-\gamma)^{2} \frac{\sigma^{2}}{2}}\left(1-p+p(1-b)^{1-\gamma}\right)$. The function $g$ is increasing if $\gamma>1$ and is decreasing if $\gamma<1$. This equation can be analyzed using standard tools from Stokey, Lucas and Prescott (1989). Define $\xi \stackrel{\text { def }}{=} \max _{\underline{p} \leq p \leq \bar{p}} g(p)$. To prove the result, we need to assume that $\xi<1 .^{8}$

Proposition 1 Assume that $\xi<1$. Then there exists a unique solution $q^{*}$ to equation (1). Moreover, $q^{*}$ is nondecreasing if $g$ is nondecreasing and is nonincreasing if $g$ is nonincreasing.

Proof. Define $B$ the set of continuous (and thus bounded) functions mapping $[\underline{p}, \bar{p}]$ into $\mathbb{R}^{+}$. Define the operator $T: B \rightarrow B$, which maps a function $q \in B$ into a new function $\widetilde{q} \in B$, defined by the

\footnotetext{
${ }^{6}$ Note that this conditional expectation is an integral over three random variables: (1) the business cycle shock $\varepsilon_{t+1}$, which is $N\left(0, \sigma^{2}\right),(2)$ the realization of the disaster, which is a binomial variable parametrized by $p_{t}$, (3) the probability of disaster next period $p_{t+1}$, which is drawn according to the Markov process $F$ given $p_{t}$. The assumptions above imply that these three variables are independent conditional on $p_{t}$, which is why the computation of the integral is simple.

${ }^{7}$ An extension of the disaster model would have positive as well as negative disasters, thus creating a pure precautionary savings effect. However, diminishing marginal utility implies that the positive disasters typically do not matter much.

${ }^{8}$ The assumption that $\xi<1$ ensures that the price of a consumption claim is finite; see section 4 for some numerical experiments to gauge its empirical realism. Because it is only a sufficient condition for prices to be finite, it could be that prices are indeed finite but the condition is violated, which would invalidate my proofs. It might be possible however to extend the proofs to allow for a weaker condition (e.g. looking for a fixed point in a different functional space).
} 
right-hand-side of (1), i.e.:

$$
\widetilde{q}(p)=(T q)(p)=g(p) \int_{\underline{p}}^{\bar{p}}\left(q\left(p^{\prime}\right)+1\right) d F\left(p^{\prime} \mid p\right)=g(p)+g(p) \int_{\underline{p}}^{\bar{p}} q\left(p^{\prime}\right) d F\left(p^{\prime} \mid p\right) .
$$

Since the Markov process $F$ has the Feller property, $T$ indeed maps $B$ into $B$. Next we show that $T$ is a contraction. To see this, pick any two $q_{1}, q_{2} \in B$, then for any $p \in[\underline{p}, \bar{p}]$ :

$$
\begin{aligned}
\left(T q_{1}\right)(p)-\left(T q_{2}\right)(p) & =g(p) \int_{\underline{p}}^{\bar{p}}\left(q_{1}\left(p^{\prime}\right)-q_{2}\left(p^{\prime}\right)\right) d F\left(p^{\prime} \mid p\right), \\
\left|\left(T q_{1}\right)(p)-\left(T q_{2}\right)(p)\right| & \leq \xi \int_{\underline{p}}^{\bar{p}}\left|\left(q_{1}\left(p^{\prime}\right)-q_{2}\left(p^{\prime}\right)\right)\right| d F\left(p^{\prime} \mid p\right), \\
& \leq \xi\left\|q_{1}-q_{2}\right\|_{\infty},
\end{aligned}
$$

hence $\sup _{[\underline{p}, \bar{p}]}\left|\left(T q_{1}\right)(p)-\left(T q_{2}\right)(p)\right|=\left\|T q_{1}-T q_{2}\right\|_{\infty} \leq \xi\left\|q_{1}-q_{2}\right\|_{\infty}$, where $\|f\|_{\infty}=\sup _{x \in[\underline{p}, \bar{p}]}|f(x)|$ is the sup norm. Since $\xi<1$, this shows that $T$ is a contraction. ${ }^{9}$ The contraction mapping theorem implies that there exists a unique solution $q^{*}$ to the fixed point problem $T q=q$. Because the Markov process $F$ is monotone, $T$ satisfies a monotonicity property. More precisely, if $g$ is nondecreasing, then $T$ maps nondecreasing functions into nondecreasing functions; and if $g$ is nonincreasing, then $T$ maps nonincreasing functions into nonincreasing functions. This can be seen from (2). For instance if $g$ is nondecreasing: we know that if $q$ is nondecreasing, the function $p \rightarrow \int_{\underline{p}}^{\bar{p}} q\left(p^{\prime}\right) d F\left(p^{\prime} \mid p\right)$ is nondecreasing; because both $g$ and $q$ are nonnegative and nondecreasing, the product $g(p) \int_{0}^{1} q\left(p^{\prime}\right) d F\left(p^{\prime} \mid p\right)$ is nondecreasing, and thus $g(p)+g(p) \int_{0}^{1} q\left(p^{\prime}\right) d F\left(p^{\prime} \mid p\right)$ is nondecreasing. Because the set of nondecreasing (resp. nonincreasing) functions is closed under the sup norm, this result implies that the fixed point $q^{*}$ is nondecreasing if $g$ is nondecreasing and is nonincreasing if $g$ is nonincreasing (Theorem 4.7 in Stokey, Lucas and Prescott (1989)).

We are now in position to compute the expected return on equity. Given the definition $E_{t} R_{t+1}^{e}=$ $E_{t}\left(P_{t+1}+C_{t+1}\right) / P_{t}$, and our assumptions regarding the process of shocks, we have:

$$
E R^{e}(p)=E_{t}\left(\frac{C_{t+1}}{C_{t}}\right) E_{p^{\prime} \mid p}\left(\frac{q\left(p^{\prime}\right)+1}{q(p)}\right) .
$$

The Euler equation (1) implies that $E_{p^{\prime} \mid p}\left(\frac{q\left(p^{\prime}\right)+1}{q(p)}\right)=\frac{1}{g(p)}$, hence:

$$
E R^{e}(p)=\frac{e^{\mu+\frac{\sigma^{2}}{2}}(1-p+p(1-b))}{\beta e^{(1-\gamma) \mu+(1-\gamma)^{2} \frac{\sigma^{2}}{2}}\left(1-p+p(1-b)^{1-\gamma}\right)} .
$$

Rearranging and taking logs yields:

$$
\log E R^{e}(p)=\gamma \mu-\frac{\gamma^{2} \sigma^{2}}{2}+\gamma \sigma^{2}-\log \beta+\log \left(\frac{1-p+p(1-b)}{1-p+p(1-b)^{1-\gamma}}\right) .
$$

Again we recognize the first four terms as the i.i.d. lognormal model. The last term, which varies over time with $p$, is decreasing in $p$, as can be easily verified by taking derivatives. A higher probability of disaster reduces expected growth, increases the risk premium, and reduces the risk-free rate, and the total effect is to reduce the expected return on equity.

\footnotetext{
${ }^{9}$ When $g$ is increasing, we can alternatively use the Blackwell sufficient conditions (see Stokey, Lucas and Prescott (1989), chapter 4) to establish this result, but when $g$ is decreasing the Blackwell sufficient conditions do not hold.
} 
The log equity premium is finally obtained as:

$$
\log \frac{E R^{e}(p)}{R^{f}(p)}=\gamma \sigma^{2}+\log \frac{(1-p+p(1-b))\left(1-p+p(1-b)^{-\gamma}\right)}{\left(1-p+p(1-b)^{1-\gamma}\right)} .
$$

Taking derivatives in this expression shows that this is an increasing function of $p$ when $p$ is small enough. ${ }^{10}$ This is intuitive: when the probability of disaster of high, the risk is high, and the equity premium is high. The following proposition summarizes the results:

Proposition 2 Assume that the Markov process $F$ is monotone and satisfies the Feller property, and that $\max _{p \in[p, \bar{p}]} \beta e^{(1-\gamma) \mu+(1-\gamma)^{2} \frac{\sigma^{2}}{2}}\left(1-p+p(1-b)^{1-\gamma}\right)<1$. Then, (a) the risk-free rate and the expected equity return are decreasing in $p$; (b) the price-dividend ratio is increasing in $p$ if and only if $\gamma>1 ;(c)$ for $p$ small enough, the equity premium is increasing in $p$.

It is interesting to relate these results to the empirical evidence outlined in the introduction. In the data, $\operatorname{Cov}_{t}\left(P_{t} / D_{t}, E_{t} R_{t+1}^{e}\right)<0$ and $\operatorname{Cov}_{t}\left(P_{t} / D_{t}, E_{t} R_{t+1}^{e}-R_{t+1}^{f}\right)<0$. Proposition 2 implies that, whatever the value of $\gamma$, the model cannot match both facts. If $\gamma>1$, then the equity premium and the P-D ratio are both increasing in $p$, hence a high P-D ratio forecasts a high excess stock return, contrary to the data. (The fact that a high P-D ratio corresponds to a high probability of disaster $p$ is also counterintuitive.) If $\gamma<1$, then the P-D ratio and the equity return are both decreasing in $p$, and hence a high P-D ratio forecasts a high equity return, contrary to the data. The reason why the disaster model generates these counterfactual implications is that it predicts large variations in risk-free interest rates: the risk-free rate moves by more than the equity risk premium, so that the expected equity return and the equity risk premium move in opposite directions.

It is straightforward to allow for leverage, using the standard formulation $D_{t}=C_{t}^{\lambda}$, where $\lambda$ measures the degree of leverage. The formulas above need to be modified: replace the factor $(1-b)^{1-\gamma}$ by $(1-b)^{\lambda-\gamma}$, and the factor $(1-b)$ by $(1-b)^{\lambda}$. As a result, the P-D ratio is increasing in $p$ if and only if $\gamma>\lambda$. The equity premium is now

$$
\log \frac{E R^{e}(p)}{R^{f}(p)}=\gamma \lambda \sigma^{2}+\log \frac{\left(1-p+p(1-b)^{\lambda}\right)\left(1-p+p(1-b)^{-\gamma}\right)}{\left(1-p+p(1-b)^{\lambda-\gamma}\right)},
$$

but the fundamental conundrum remains: neither $\gamma>\lambda$ nor $\lambda>\gamma$ allows the model to generate both the stock return and the excess stock return predictability.

We can also extend the results to the realistic case where government bonds are not risk-free during disasters. For instance, if the government defaults by an amount $1-r$ in disasters (i.e. $r$ is the recovery rate), it is easy to show that the log expected return on the government bond is:

$$
\log E R^{b}(p)=-\log \beta+\gamma \mu-\frac{\gamma^{2} \sigma^{2}}{2}+\log \left(\frac{1-p+p \times r}{1-p+p(1-b)^{-\gamma} r}\right)
$$

a formula which incorporates the special case of a truly risk-free asset $(r=1)$. The equity risk premium is then:

$$
\log \frac{E R^{e}(p)}{E R^{b}(p)}=\gamma \sigma^{2}+\log \frac{\left(1-p+p(1-b)^{-\gamma} r\right)(1-p+p(1-b))}{\left(1-p+p(1-b)^{1-\gamma}\right)(1-p+p \times r)},
$$

\footnotetext{
${ }^{10}$ Because disasters are a binomial variable, the uncertainty is highest for intermediate values of $p$, and hence the risk premium is not increasing over the entire range of values: if $p$ is large enough, a further increase reduces the uncertainty and thus the risk premium. This remark is not important in practice because disasters are always calibrated as rare events.
} 
but the results are unchanged as long as $r>1-b$, so that government bonds are less risky than equities during disasters.

Rather than having the probability of disaster $p$ change over time, one may assume that it is the size of disasters $b$ that changes over time, i.e.

$$
\begin{aligned}
\Delta \log C_{t+1} & =\mu+\sigma \varepsilon_{t+1}, \text { with probability } 1-p \\
\Delta \log C_{t+1} & =\mu+\sigma \varepsilon_{t+1}+\log \left(1-b_{t}\right), \text { with probability } p
\end{aligned}
$$

If we make the same assumptions for $b$ as the ones we did for $p$ above, it is straightforward to prove the following analogous result. More precisely, define $g(b)=\beta e^{(1-\gamma) \mu+(1-\gamma)^{2} \frac{\sigma^{2}}{2}}\left(1-p+p(1-b)^{1-\gamma}\right)$. Assume $b$ follows a Markov process with support $[\underline{b}, \bar{b}]$ with transition function $F$, and assume that the Markov process is monotone and satisfies the Feller property, and that the independence assumptions hold.

Proposition 3 Assume $\max _{\underline{b} \leq b \leq \bar{b}} g(b)<1$. Then there exists a unique solution $q^{*}$ to the functional equation defining the price-dividend ratio. Moreover, (a) the risk-free rate and the expected equity return are decreasing in $b$; (b) the price-dividend ratio is increasing in $b$ if and only if $\gamma>1$; (c) if $p$ is small enough, the equity premium is increasing in $b$.

This extension thus does not resolve the previously noted conundrum. Finally, a last extension is to only allow the size of dividend disasters to change over time. Assume, then, that the stochastic processes for consumption and dividends satisfy:

$$
\begin{aligned}
\Delta \log C_{t+1} & =\mu+\sigma \varepsilon_{t+1}, \\
\text { and } \Delta \log D_{t+1} & =\mu+\sigma \varepsilon_{t+1}, \text { with probability } 1-p ; \\
\text { or } \Delta \log C_{t+1} & =\mu+\sigma \varepsilon_{t+1}+\log (1-b), \\
\text { and } \Delta \log D_{t+1} & =\mu+\sigma \varepsilon_{t+1}+\log \left(1-d_{t}\right), \text { with probability } p,
\end{aligned}
$$

where $d_{t}$ follows a monotone Feller Markov process with support $[\underline{d}, \bar{d}]$, and the independence assumptions hold. Let $g(d)=\beta e^{(1-\gamma) \mu+(1-\gamma)^{2} \frac{\sigma^{2}}{2}}\left(1-p+p(1-d)(1-b)^{-\gamma}\right)$. In this case, $g$ is always nonincreasing.

Proposition 4 Assume $\max _{\underline{d} \leq d \leq \bar{d}} g(d)<1$. Then there exists a unique solution $q^{*}$ to the functional equation defining the price-dividend ratio. Moreover, (a) the risk-free rate is constant, (b) the pricedividend ratio is decreasing in $d$, (c) the expected equity return and equity premium are increasing in $d$.

Hence, this model is the only variation consistent with both pieces of evidence on predictability summarized in the introduction. It also generates the intuitive result that a high expected size of disaster leads to a low P-D ratio. However, it is unclear if this assumption (that the size of the potential dividend disaster changes over time, but the size of the potential consumption disaster does not) is empirically reasonable. In general equilibrium, consumption and dividends must be somewhat related; 
at long horizons especially, we expect dividends and consumption to be cointegrated, so even if we think that dividends or earnings may fall a lot if there is a disaster (e.g., Longstaff and Piazzesi (2004)), they would surely increase back after a while. ${ }^{11}$ Second, as explained in the introduction, the empirical finance literature suggests that risk premia on various assets are correlated. Generating this pattern will require that the size of dividend disasters on these various assets move over time and are correlated. This seems somewhat ad-hoc, and certainly less appealing than a unifying explanation through consumption, which affects all the assets - i.e. the price of consumption risk changes over time.

While this section has shown that the model cannot reproduce these findings qualitatively, the quantitative magnitude of these failures is not readily apparent. Section 4 presents some numerical examples.

\section{Epstein-Zin preferences and i.i.d. probability of disaster}

Since the failure of the disaster model in Proposition 2 is due to the fact that interest rates vary too much, it seems useful to allow for Epstein-Zin utility so as to separate intertemporal elasticity of substitution (IES) from risk aversion, and to use the IES parameter to reduce the volatility of the risk-free rate. Utility is defined recursively as

$$
V_{t}=\left((1-\beta) C_{t}^{1-\alpha}+\beta E_{t}\left(V_{t+1}^{1-\theta}\right)^{\frac{1-\alpha}{1-\theta}}\right)^{\frac{1}{1-\alpha}} .
$$

With these preferences, the IES is $1 / \alpha$ and the risk aversion to a static gamble is $\theta$. When $\theta=\alpha$, or if there is no risk, these preferences collapse to the familiar case of expected utility. In general we cannot reduce compound lotteries, so that the intertemporal composition of risk matters: the agent prefers an early resolution of uncertainty if $\theta>\alpha$. The stochastic discount factor is:

$$
M_{t+1}=\beta\left(\frac{C_{t+1}}{C_{t}}\right)^{-\alpha}\left(\frac{V_{t+1}}{E_{t}\left(V_{t+1}^{1-\theta}\right)^{\frac{1}{1-\theta}}}\right)^{\alpha-\theta} .
$$

I assume that the stochastic process for the endowment is the same as in Proposition 2. This section analyzes the case of an i.i.d. probability of disaster: at each date $t$, a new probability $p_{t}$ that a disaster occurs at time $t+1$ is drawn from the same distribution $F$. This is the only case which appears to be solvable with pencil and paper. The next section studies numerically the case of a persistent probability of disaster.

Proposition 5 Assume that the disaster probability is i.i.d., i.e. $F\left(p_{t+1} \mid p_{t}\right)=F\left(p_{t+1}\right)$. Then (a) if $\theta \geq 1$, the $P$ - $D$ ratio is increasing in $p$ if and only if $\alpha>1$; (b) if $\theta \geq 1$, the risk-free rate is decreasing in $p$ for $p$ small enough, and the expected equity return is decreasing in $p$ if $\alpha>1$, but can be increasing or decreasing in $p$ if $\alpha<1$; (c) the equity premium is always increasing in $p$, for $p$ small enough.

Proof. First, rewrite the utility recursion as:

$$
\frac{V_{t}}{C_{t}}=\left(1-\beta+\beta E_{t}\left(\left(\frac{V_{t+1}}{C_{t+1}}\right)^{1-\theta}\left(\frac{C_{t+1}}{C_{t}}\right)^{1-\theta}\right)^{\frac{1-\alpha}{1-\theta}}\right)^{\frac{1}{1-\alpha}} .
$$

\footnotetext{
${ }^{11}$ See Gourio (2008) for a simple analysis of the dynamics after disaster.
} 
The state variable is the probability of a disaster $p$. Let $g\left(p_{t}\right)=V_{t} / C_{t}$. The two random variables $\frac{C_{t+1}}{C_{t}}$ and $\frac{V_{t+1}}{C_{t+1}}$ are independent, hence $g$ satisfies the functional equation:

$$
g(p)^{1-\alpha}=1-\beta+\beta\left(E g\left(p^{\prime}\right)^{1-\theta}\right)^{\frac{1-\alpha}{1-\theta}}\left(1-p+p(1-b)^{1-\theta}\right)^{\frac{1-\alpha}{1-\theta}} e^{(1-\alpha) \mu+(1-\alpha)(1-\theta) \frac{\sigma^{2}}{2}},
$$

where the expectation is over $p^{\prime}$ next period; given the iid assumption, this expectation is independent of $p$. The price-dividend ratio satisfies $P_{t} / C_{t}=q\left(p_{t}\right)$ with:

$$
\begin{aligned}
& q(p)=E_{t}\left(M_{t+1} \frac{C_{t+1}}{C_{t}}\left(1+\frac{P_{t+1}}{C_{t+1}}\right)\right), \\
& q(p)=E_{t}\left(\beta\left(\frac{C_{t+1}}{C_{t}}\right)^{1-\alpha}\left(\frac{V_{t+1}}{E_{t}\left(V_{t+1}^{1-\theta}\right)^{\frac{1}{1-\theta}}}\right)^{\alpha-\theta}\left(1+\frac{P_{t+1}}{C_{t+1}}\right)\right) .
\end{aligned}
$$

Straightforward computations yield:

$$
q(p)=\beta e^{(1-\alpha) \mu+(1-\theta)(1-\alpha) \frac{\sigma^{2}}{2}}\left(1-p+p(1-b)^{1-\theta}\right)^{\frac{1-\alpha}{1-\theta}} \frac{E\left(g\left(p^{\prime}\right)^{\alpha-\theta}\left(1+q\left(p^{\prime}\right)\right)\right)}{E\left(g\left(p^{\prime}\right)^{1-\theta}\right)^{\frac{\alpha-\theta}{1-\theta}}} .
$$

The expectation on the right-hand side is independent of $p$, given the iid assumption. Hence, if $\theta>1$, the price-dividend ratio $q(p)$ is increasing in $p$ if and only if $(1-\alpha) /(1-\theta)>0$ i.e. $1-\alpha<0$ or $\alpha>1$, i.e. an elasticity of substitution less than unity.

The expected equity return is:

$$
\begin{aligned}
E_{t} R_{t+1}^{e}(p) & =E_{t}\left(\frac{q\left(p_{t+1}\right)+1}{q(p)} \frac{C_{t+1}}{C_{t}}\right) \\
& =\frac{E\left(q\left(p^{\prime}\right)\right)+1}{q(p)}(1-p+p(1-b)) e^{\mu+\frac{\sigma^{2}}{2}} \\
& =\frac{(1-p+p(1-b)) e^{\mu+\frac{\sigma^{2}}{2}}}{\beta e^{(1-\alpha) \mu+(1-\theta)(1-\alpha) \frac{\sigma^{2}}{2}}\left(1-p+p(1-b)^{1-\theta}\right)^{\frac{1-\alpha}{1-\theta}}} \frac{E\left(q\left(p^{\prime}\right)+1\right) E\left(g\left(p^{\prime}\right)^{1-\theta}\right)^{\frac{\alpha-\theta}{1-\theta}}}{E\left(g\left(p^{\prime}\right)^{\alpha-\theta}\left(1+q\left(p^{\prime}\right)\right)\right)} .
\end{aligned}
$$

Hence,

$$
\log E R^{e}(p)=\mathrm{constant}-\log \beta+\alpha \mu+\frac{\sigma^{2}}{2}(1-(1-\theta)(1-\alpha))+\log \frac{(1-p+p(1-b))}{\left(1-p+p(1-b)^{1-\theta}\right)^{\frac{1-\alpha}{1-\theta}}}
$$

Note that in contrast to Proposition 2, this need not be decreasing in $p$. Taking derivatives, one can see that there are two effects. If $\alpha>1$, the total effect is negative, but if $\alpha<1$, it can be positive.

The risk-free rate is:

$$
\begin{aligned}
R^{f}(p) & =\frac{1}{E_{t}\left(M_{t+1}\right)}=\frac{E_{t}\left(\left(\frac{V_{t+1}}{C_{t+1}}\right)^{1-\theta}\left(\frac{C_{t+1}}{C_{t}}\right)^{1-\theta}\right)^{\frac{\alpha-\theta}{1-\theta}}}{\beta E_{t}\left(\left(\frac{C_{t+1}}{C_{t}}\right)^{-\theta}\left(\frac{V_{t+1}}{C_{t+1}}\right)^{\alpha-\theta}\right)}, \\
& =\frac{\left(1-p+p(1-b)^{1-\theta}\right)^{\frac{\alpha-\theta}{1-\theta}} e^{(\alpha-\theta) \mu+(\alpha-\theta)(1-\theta) \frac{\sigma^{2}}{2}} E\left(g\left(p^{\prime}\right)^{1-\theta}\right)^{\frac{\alpha-\theta}{1-\theta}}}{\beta e^{-\theta \mu+\theta^{2} \frac{\sigma^{2}}{2}}\left(1-p+p(1-b)^{-\theta}\right) E\left(g\left(p^{\prime}\right)^{\alpha-\theta}\right)} .
\end{aligned}
$$

Hence,

$$
\log R^{f}(p)=\text { constant }-\log \beta+\alpha \mu+(\alpha-\theta-\theta \alpha) \frac{\sigma^{2}}{2}+\log \frac{\left(1-p+p(1-b)^{1-\theta}\right)^{\frac{\alpha-\theta}{1-\theta}}}{\left(1-p+p(1-b)^{-\theta}\right)} .
$$

We want to prove that $p \rightarrow \frac{\left(1-p+p(1-b)^{1-\theta}\right)^{\frac{\alpha-\theta}{1-\theta}}}{\left(1-p+p(1-b)^{-\theta}\right)}$ is decreasing for any $\alpha$. The derivative has the sign of

$$
\frac{\alpha-\theta}{1-\theta} \frac{\left((1-b)^{1-\theta}-1\right)\left(1-p+p(1-b)^{-\theta}\right)}{\left(1-p+p(1-b)^{1-\theta}\right)}-\left((1-b)^{-\theta}-1\right) \text {. }
$$


To prove that this is positive, for any $\alpha$, it is sufficient to show that

$$
\frac{-\theta}{1-\theta}\left((1-b)^{1-\theta}-1\right)\left(1-p+p(1-b)^{-\theta}\right)<\left((1-b)^{-\theta}-1\right)\left(1-p+p(1-b)^{1-\theta}\right) .
$$

By continuity, this is true for small $p$ if and only if

$$
\frac{(1-b)^{1-\theta}-1}{1-\theta}>\frac{(1-b)^{-\theta}-1}{-\theta},
$$

which is true since it is easy to verify that the map $\alpha \rightarrow \frac{x^{\alpha}-1}{\alpha}$ is increasing in $\alpha$ for $\alpha<0$ and $0<x<1$.

Finally, the log equity premium is:

$$
\begin{aligned}
\log \frac{E R^{e}(p)}{R^{f}(p)}= & \text { constant }+\frac{\sigma^{2}}{2}(1-(1-\theta)(1-\alpha))-(\alpha-\theta-\theta \alpha) \frac{\sigma^{2}}{2} \\
& +\log \frac{(1-p+p(1-b))\left(1-p+p(1-b)^{-\theta}\right)}{\left(1-p+p(1-b)^{1-\theta}\right)} \\
= & \text { constant }+\theta \sigma^{2}+\log \frac{(1-p+p(1-b))\left(1-p+p(1-b)^{-\theta}\right)}{\left(1-p+p(1-b)^{1-\theta}\right)} .
\end{aligned}
$$

As in Section 2, it is easy to see by taking derivatives that this term is increasing in $p$ for small $p$.

The main difference with the case of power utility studied in the previous section, is that the expected equity return need not be decreasing in $p$ if the IES $1 / \alpha$ is larger than unity. Hence, this analysis reveals that introducing Epstein-Zin preferences can potentially resolve the puzzle raised above: a calibration with an IES above unity can generate that an increase in the probability of disaster lowers prices, increases the equity premium and the expected equity return while lowering the risk-free rate. The next section examines the quantitative success of this model.

Note that the computations above can be extended for leverage and government default. The more general formulas are

$$
q(p)=\beta e^{(\lambda-\alpha) \mu+\left((\lambda-\theta)^{2}+(1-\theta)(\theta-\alpha)\right) \frac{\sigma^{2}}{2}} \frac{\left(1-p+p \times(1-b)^{\lambda-\theta}\right)}{\left(1-p+p \times(1-b)^{1-\theta}\right)^{\frac{\alpha-\theta}{1-\theta}}} \frac{E\left(g\left(p^{\prime}\right)^{\alpha-\theta}\left(1+q\left(p^{\prime}\right)\right)\right)}{E\left(g\left(p^{\prime}\right)^{1-\theta}\right)^{\frac{\alpha-\theta}{1-\theta}}},
$$

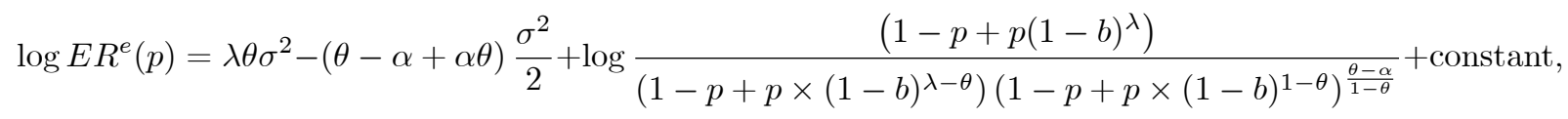
$\log E R^{b}(p)=-\log \beta+\alpha \mu+(\alpha-\theta-\theta \alpha) \frac{\sigma^{2}}{2}+\log \frac{(1-p+p r)\left(1-p+p(1-b)^{1-\theta}\right)^{\frac{\alpha-\theta}{1-\theta}}}{\left(1-p+p(1-b)^{-\theta} r\right)}+$ constant.

\section{Numerical Results}

This section illustrates the qualitative results of Sections 2 and 3, and relaxes some assumptions which were necessary to obtain these qualitative results. Finally I consider in detail the most promising case of Epstein-Zin utility with persistent probability of disaster. When the probability of disaster $p$ follows a Markov chain, the model can be solved numerically with standard techniques. ${ }^{12}$

\subsection{Calibration of the model}

Most of the parameters are drawn from Barro (2006). The discount factor is $\beta=0.97$, the trend growth rate is $\mu=0.025$, the standard deviation of the normally distributed shock is $\sigma=0.02$, and risk aversion

\footnotetext{
${ }^{12}$ The details and code are are available on my website: http://people.bu.edu/fgourio/research.html.
} 
is $\theta=4$. I will present the results for various intertemporal elasticities of substitution $1 / \alpha$. The disaster size is $b=0.43$, and it is assumed that government bonds default by an amount $1-b$ with probability 0.4 when there is a disaster. ${ }^{13}$ The process for consumption growth is

$$
\begin{aligned}
\Delta \log C_{t+1} & =\mu+\sigma \varepsilon_{t+1}, \text { with probability } 1-p_{t} \\
& =\mu+\sigma \varepsilon_{t+1}+\log (1-b), \text { with probability } p_{t}
\end{aligned}
$$

where $\varepsilon_{t+1}$ is iid $N(0,1)$. I assume that $p_{t}$ follows a two-state Markov chain, characterized by the two values $p_{h}$ and $p_{l}$ and the transition matrix:

$$
Q=\left(\begin{array}{cc}
1-\pi & \pi \\
\pi & 1-\pi
\end{array}\right)
$$

Note that in the interest of parsimony, I assume that $Q$ is symmetric. The new parameters to calibrate are the probabilities of disaster in the high and low state, $p_{h}$ and $p_{l}$, as well as the transition probability $\pi$. To calibrate these parameters, I keep the average (over time) of the probability of disaster equal to $1.7 \%$, as in Barro (2006): hence $p_{h}=.017+\varepsilon$ and $p_{l}=.017-\varepsilon$, and this leaves two degrees of freedom: $\pi$ and $\varepsilon$. There is little guidance for these parameters. I start by setting $\varepsilon=.01$ and $\pi=.1$ and will later pick these parameters to maximize the fit of the model.

Note that given the values for $\beta, \gamma, \mu, b$, and $\sigma$, the assumption that $\xi<1$ is equivalent here to $p_{h}<0.03009$. i.e. the probability of disaster is always less than $3 \%$ per year. This is much above the average value of $p$ estimated by Barro (2006), which is $1.7 \%$, but of course some calibrations could require that $p$ sometimes wanders to $3 \%$ or higher, in which case my sufficient condition would be violated. ${ }^{14}$ For a higher level of risk aversion, the maximum $p$ allowable will be smaller, e.g. if risk aversion is 6 , it is approximately $1.37 \%$, and if risk aversion is 3 , it is $4.65 \%$.

\subsection{Illustration of the Qualitative Results}

I concentrate on the following moments: the unconditional means and standard deviation of the equity return and the government bond return, the standard deviation of dividend growth and the pricedividend ratio, and the slope coefficients $\beta_{R^{e}}$ and $\beta_{R^{e} R^{f}}$ in the regressions presented in the introduction (equity return and equity excess return on dividend yield respectively). To make the mechanics of the model transparent, Table 1 presents the model-implied P-D ratios and expected returns and excess returns conditional on each state. State 1 has a low probability of disaster next period and state 2 has a high probability of disaster next period. Table 2 computes the moments implied by the model. Table 3 presents the moments in a sample in which no disaster occurred.

Consider first the case of expected utility analyzed in section 2 : we set $\alpha=1 / \theta=.25$. The results are reported in row 1. This model can generate large variations in the equity premium (from $1.77 \%$ in the low probability state to $5.08 \%$ in the high probability state), but it also implies large changes in

\footnotetext{
${ }^{13}$ Barro (2006) actually uses the historical distribution of disaster sizes, but the equity premium he obtains is the same as the one implied by a single $b=.43$.

${ }^{14}$ Numerically, even if $p$ is sometimes higher than this critical value, prices are still finite as long as $p$ is not too high "too often". I did not find an example of finite prices where the analytical results of sections 2 and 3 did not hold.
} 
the expected bond return ( $8.25 \%$ in the low probability state and $-4.59 \%$ in the high probability state). As proved in Section 2, the regression coefficients $\beta_{R^{e}}$ and $\beta_{R^{e} R^{f}}$ have opposite signs, contrary to the data. As argued in section 2, the government bond return -which is not purely risk-free in this modelis fairly volatile $(6.78 \%$ vs. $4.36 \%$ in the data) and the price-dividend ratio is not volatile enough (.183 vs. .415).

Row 2 adds leverage to the model. Leverage allows to match more closely the volatility of dividend growth. I follow the standard formulation: $\Delta \log D_{t}=\lambda \Delta \log C_{t}$, and I set $\lambda=2.5 .{ }^{15}$ The volatility of dividends jumps from $2.05 \%$ to $5.33 \%$ without disasters (6.05\% to $11.07 \%$ with disasters). This leads to higher risk premia and lower P-D ratios, but does not affect the signs of the regression coefficients, since the leverage $\lambda$ is less than risk aversion $\gamma$. Moreover, the volatility in prices is even lower. Of course, bond returns are unaffected by leverage.

It is easy to check now if the independence assumption (i.e., the new draw $p_{t+1}$ is independent on whether there was a disaster at $t+1$, conditional on $p_{t}$ ) affects the result quantitatively. Row 3 assumes that if there is a disaster, the probability of drawing a high $p$ is high, and row 4 considers the opposite case. Formally, the matrix $Q$ is different in the disaster state:

$$
Q=\left(\begin{array}{cc}
1-\pi^{\prime} & \pi^{\prime} \\
\pi^{\prime \prime} & 1-\pi^{\prime \prime}
\end{array}\right),
$$

with $\pi^{\prime}>\pi$ and $\pi^{\prime \prime}<\pi$ for row 3 (and the opposite for row 4): if there is a disaster, the probability of switching from low to high probability of disaster is higher than usual, and the probability of switching from high to low probability of disaster is lower than usual. I set $\pi^{\prime}=.15$ and $\pi^{\prime \prime}=.05$ to illustrate this effect. The changes are relatively minor: the expected equity return is $5.26 \%$ in row $1,5.42 \%$ in row 3 and $5.09 \%$ in row 4 . The regression coefficients, the volatility of P-D ratios, and the volatility of the government bond return are also only slightly affected.

Row 5 turns to the case of Epstein-Zin utility and i.i.d. probabilities of disasters: $\pi=.5$. For this example, I set $\alpha=.67$, corresponding to an elasticity of substitution of 1.5. As discussed in section 3, the model now generates the correct sign for the two regressions. However, the magnitude is off, since the expected equity return varies much less than the equity premium - the price-dividend ratio is not volatile enough. This turns out to be mostly due to leverage. ${ }^{16}$

\subsection{Epstein-Zin utility and persistent probability of disaster}

I now evaluate the model by picking parameters to maximize its fit. Given that the model does reasonably well for the average risk-free rate and equity premium (since on average this is the disaster model of Barro (2006)), I pick $\pi$ and $\varepsilon$ to match the standard deviation of the log price-dividend ratio and the regression coefficient of excess equity return on the dividend yield in a sample without disasters. For these experiments I set the leverage parameter to the standard value $\lambda=3$, implying that dividends fall by $80 \%$ during disasters. This is consistent with the view of Longstaff and Piazzesi (2004) who argue that

\footnotetext{
${ }^{15} \mathrm{~A}$ higher value of $\lambda$, such as the standard value of 3 , leads to infinite prices for these parameter values.

${ }^{16}$ If the elasticity of substitution is unity, the price-dividend ratio is constant, as can be seen from the formulas of section 3. Adding leverage introduces some variation in the price-dividend ratio however.
} 
dividends were "obliterated" during the Great Depression. Matching the two targets without leverage is impossible: the variation in the price-dividend ratio is much too low.

Row 6 reports the results of this experiment when $\alpha$ is set equal to .67. The best fitting parameters are $\varepsilon=.169$, so that the probability of disaster oscillates between almost $0 \%$ and $3.4 \%$, and the transition probability is $\pi=4.26 \%$. Row 7 reports the results when $\alpha=1$. In this case the best fit is obtained for $\varepsilon=.160$ and $\pi=3.09 \%$. Both of these calibrations yield attractive results: the volatility of equity returns is high, the volatility of the bond return is low, the regression coefficients are in the right ballpark. Dividend volatility is high, given the high leverage, but this may be consistent with the data. The main problem is that the average equity premium is too high: to generate enough return predictability, the model needs to have very large equity premia on average. A calibration with a lower risk aversion coefficient can partially mitigate this issue.

Is this calibration reasonable? This crucial question is hard to answer, since the success of this calibration is solely driven by the large and persistent variation in the disaster probability, which is unobservable.

\section{Conclusions}

One purpose of this note is to show a simple setup in which it is possible to obtain exact analytical results in asset pricing models with time-varying risk premia and time-varying interest rates, without assuming log-linearity or log-normality. The critical assumption is that the state variable determining risk premia is conditionally independent of the variable(s) determining dividend growth and consumption growth. As a result, this state variable itself is not priced, which simplifies the analysis.

Substantially, my results suggest that it is difficult for the disaster model to fit the facts on predictability of stock returns and excess stock returns. With Epstein-Zin utility, the model can fit the facts qualitatively, and to some extent quantitatively, if we allow for a highly variable probability of disaster, leverage, and an IES above unity. However, an IES above unity diminishes markedly the equity premium implied by the model if disasters are not fully permanent, as explained in Gourio (2008). 


\section{References}

[1] Ang, Andrew and Geert Bekaert, 2007: "Stock Return Predictability: Is it There?", Review of Financial Studies, 20(3):651-707.

[2] Bansal Ravi and Amir Yaron, 2004: "Risks for the Long Run: A Potential Explanation of Asset Pricing Puzzles", Journal of Finance 59: 1481-1509.

[3] Barro Robert, 2006: "Rare Disasters and Asset Markets in the Twentieth Century", Quarterly Journal of Economics 121:823-866.

[4] Boudoukh, Jacob, Matthew Richardson and Robert Whitelaw, 2008: "The Myth of Long-Horizon Predictability", Review of Financial Studies, Forthcoming.

[5] Campbell, John, 1996: "Understanding Risk and Return", Journal of Political Economy 104(2):298345.

[6] Campbell, John, and John Cochrane, 1999: "By Force of Habit: A Consumption-Based Explanation of Aggregate Stock Market Behavior", Journal of Political Economy 107(2):205-251.

[7] Cochrane John, 2008: "The Dog that did not Bark", Review of Financial Studies, forthcoming.

[8] Gabaix, Xavier, 2007: "Variable Rare Disasters: An Exactly Solved Framework for Ten Puzzles in Macro-Finance". Mimeo, NYU.

[9] Gabaix, Xavier, 2007b: "Linearity-Generating Processes: A Modelling Tool Yielding Closed Forms for Asset Prices". Mimeo, NYU.

[10] Gourio, Francois, 2008: "Disasters and Recoveries", American Economic Review Papers and Proceedings, 98(2): 68-73.

[11] Goyal, Amit, and Io Welch, 2007: "A Comprehensive Look at the Empirical Performance of Equity Premium Prediction", Review of Financial Studies, 2007.

[12] Longstaff, Francis, and Monika Piazzesi, 2004: "Corporate earnings and the equity premium", Journal of Financial Economics, 74(3):401-421.

[13] Mehra, Rajnish and Edward Prescott, 1985: "The Equity Premium: a Puzzle". Journal of Monetary Economics, 15:145-161.

[14] Rietz Thomas, 1988: "The Equity Premium: a Solution”, Journal of Monetary Economics 22:117131.

[15] Stambaugh, Robert, 1999: "Predictive Regressions", Journal of Financial Economics 54:375-421.

[16] Stokey, Lucas and Prescott, 1989: "Recursive Methods in Economics Dynamics", Harvard University Press.

[17] Wachter, Susan (2008). "Can time-varying risk of rare disasters explain aggregate stock market volatility?". Mimeo, Wharton. 


\begin{tabular}{l|lllll|llll|llll}
\hline \hline & \multicolumn{3}{|l|}{ Parameters } & \multicolumn{4}{|c|}{ Low probability state } & \multicolumn{4}{|l}{ High probability state } \\
Row & $I E S$ & $p_{l}$ & $p_{h}$ & $\pi$ & $\lambda$ & $P D$ & $E R^{b}$ & $E R^{e}$ & $E\left(R^{e}-E R^{b}\right)$ & $P D$ & $E R^{b}$ & $E R^{e}$ & $E\left(R^{e}-R^{b}\right)$ \\
\hline 1 & 0.250 & 0.7 & 2.7 & 0.100 & 1 & 31.4 & 8.2 & 10.0 & 1.77 & 45.25 & -4.59 & 0.49 & 5.08 \\
2 & 0.250 & 0.7 & 2.7 & 0.100 & 2.5 & 20.7 & 8.2 & 12.4 & 4.13 & 23.07 & -4.59 & 7.85 & 12.43 \\
3 & 0.250 & 0.7 & 2.7 & 0.100 & 1 & 33.4 & 8.2 & 9.95 & 1.70 & 48.52 & -4.59 & 0.31 & 4.90 \\
4 & 0.250 & 0.7 & 2.7 & 0.100 & 1 & 29.7 & 8.2 & 10.1 & 1.84 & 42.48 & -4.59 & 0.67 & 5.26 \\
5 & 1.5 & 0.7 & 2.7 & 0.500 & 1 & 32.6 & 3.2 & 4.9 & 1.72 & 32.30 & -0.39 & 4.96 & 5.35 \\
6 & 1.5 & 0.01 & 3.39 & 0.043 & 3 & 25.4 & 4.1 & 9.6 & 5.51 & 11.17 & -1.55 & 20.10 & 21.64 \\
7 & 1 & 0.1 & 3.3 & 0.031 & 3 & 25.5 & 5.4 & 10.3 & 4.96 & 11.63 & -1.98 & 17.98 & 19.96 \\
\hline \hline
\end{tabular}

Table 1: This table reports, for various parameter values, the price-dividend ratio, the expected bond return, equity return, and equity premium, in both the low probability state and the high probability state. Rows 3 and 4 allow for disasters to be correlated with the future probability of disasters.

\begin{tabular}{llllll|llllllll}
\hline \hline Row & $I E S$ & $p_{l}$ & $p_{h}$ & $\pi$ & $\lambda$ & $E R^{b}$ & $E R^{e}$ & $\sigma\left(R^{e}\right)$ & $\sigma\left(R^{b}\right)$ & $\sigma(D)$ & $\sigma(p-d)$ & $\beta_{R^{e} R^{b}}$ & $\beta_{R^{e}}$ \\
\hline \hline 1 & 0.250 & 0.7 & 2.7 & 0.100 & 1 & 1.83 & 5.26 & 14.00 & 6.78 & 6.05 & .183 & -3.43 & 9.71 \\
2 & 0.250 & 0.7 & 2.7 & 0.100 & 2.51 & 1.83 & 10.11 & 12.81 & 6.78 & 11.67 & .055 & -16.45 & 9.00 \\
3 & 0.250 & 0.7 & 2.7 & 0.100 & 1 & 1.78 & 5.09 & 14.16 & 6.78 & 6.07 & .187 & -3.37 & 10.37 \\
4 & 0.250 & 0.7 & 2.7 & 0.100 & 1 & 1.88 & 5.42 & 13.83 & 6.78 & 6.04 & .179 & -3.44 & 9.20 \\
5 & 1.5 & 0.7 & 2.7 & 0.500 & 1 & 1.41 & 4.94 & 6.26 & 2.87 & 6.05 & .005 & 126.90 & 0.75 \\
6 & 1.5 & 0.01 & 3.39 & 0.043 & 3 & 1.29 & 14.87 & 26.30 & 3.58 & 13.07 & .411 & 3.21 & 2.08 \\
7 & 1 & 0.1 & 3.3 & 0.031 & 3 & 1.69 & 14.15 & 22.66 & 4.27 & 13.07 & .392 & 3.20 & 1.63 \\
\hline \hline Data & - & - & - & - & - & 1.03 & 8.91 & 15.04 & 4.36 & 14.86 & .415 & 3.83 & 3.39 \\
\hline \hline
\end{tabular}

Table 2: This table reports, for various parameter values, the model-implied (full sample) unconditional mean and standard deviation of the equity return and the government bond return; unconditional standard deviation of dividend growth and the log price-dividend ratio; and the regression slopes of equity return and excess return on the dividend yield. The data statistics are based on the 1926-2004 sample used by Cochrane (2008). Rows 3 and 4 allow for disasters to be correlated with the future probability of disasters. 


\begin{tabular}{llllll|lllllllll}
\hline \hline Row & $I E S$ & $p_{l}$ & $p_{h}$ & $\pi$ & $\lambda$ & $E R^{b}$ & $E R^{e}$ & $\sigma\left(R^{e}\right)$ & $\sigma\left(R^{b}\right)$ & $\sigma(D)$ & $\sigma(p-d)$ & $\beta_{R^{e} R^{b}}$ & $\beta_{R^{e}}$ \\
\hline \hline 1 & 0.250 & 0.700 & 2.700 & 0.100 & 1 & 2.169 & 6.050 & 12.679 & 6.260 & 2.051 & 0.183 & -3.980 & 8.835 \\
2 & 0.250 & 0.700 & 2.700 & 0.100 & 2.5 & 2.169 & 11.547 & 6.764 & 6.260 & 5.332 & 0.055 & -19.064 & 5.769 \\
3 & 0.250 & 0.700 & 2.700 & 0.100 & 1 & 2.116 & 5.868 & 12.935 & 6.260 & 2.051 & 0.187 & -3.935 & 9.462 \\
4 & 0.250 & 0.700 & 2.700 & 0.100 & 1 & 2.222 & 6.224 & 12.434 & 6.260 & 2.051 & 0.179 & -3.983 & 8.349 \\
5 & 1.5 & 0.700 & 2.700 & 0.500 & 1 & 1.702 & 5.715 & 2.217 & 1.625 & 2.051 & 0.005 & 150.973 & 34.306 \\
6 & 1.5 & 0.010 & 3.390 & 0.043 & 3 & 1.623 & 16.474 & 23.551 & 2.551 & 6.485 & 0.411 & 3.768 & 2.750 \\
7 & 1 & 0.100 & 3.300 & 0.031 & 3 & 2.030 & 15.741 & 19.344 & 3.402 & 6.485 & 0.392 & 3.772 & 2.315 \\
\hline \hline Data & - & - & - & - & - & 1.03 & 8.91 & 15.04 & 4.36 & 14.86 & .415 & 3.83 & 3.39 \\
\hline \hline
\end{tabular}

Table 3: This table reports, for various parameter values, the model-implied unconditional mean and standard deviation of the equity return and the government bond return; unconditional standard deviation of dividend growth and the log price-dividend ratio; and regression slope of equity return and excess return on the dividend yield. These statistics are computed for a sample without disasters. The data statistics are based on the 1926-2004 sample used by Cochrane (2008). Rows 3 and 4 allow for disasters to be correlated with the future probability of disasters. 